\title{
Influence of non-conservative optical forces on the dynamics of optically trapped colloidal spheres: The fountain of probability
}

\author{
Yohai Roichman, ${ }^{1}$ Bo Sun, ${ }^{1}$ Allan Stolarski, ${ }^{2}$ and David G. Grier ${ }^{1}$ \\ ${ }^{1}$ Department of Physics and Center for Soft Matter Research, New York University, New York, NY 10003 \\ ${ }^{2} N E S T+m$, New York, NY 10002
}

(Dated: November 7, 2018)

\begin{abstract}
We demonstrate both experimentally and theoretically that a colloidal sphere trapped in a static optical tweezer does not come to equilibrium, but rather reaches a steady state in which its probability flux traces out a toroidal vortex. This non-equilibrium behavior can be ascribed to a subtle bias of thermal fluctuations by non-conservative optical forces. The circulating sphere therefore acts as a Brownian motor. We briefly discuss ramifications of this effect for studies in which optical tweezers have been treated as potential energy wells.
\end{abstract}

Most discussions of the dynamics of optically trapped particles assume at least implicitly that the forces exerted by an optical tweezer [1] are path-independent and therefore conserve mechanical energy. Optical forces due to gradients in the intensity are manifestly conservative in this sense 2]. Radiation pressure, by contrast, is not [2, 3]. The experimental studies described in this Letter demonstrate that the non-conservative component of the optical force has measurable consequences for the dynamics of optically trapped colloidal spheres. In particular, the probability density for a sphere trapped in a static optical tweezer exhibits steady-state toroidal currents, a phenomenon we call a fountain of probability. We use the Fokker-Planck formalism to explain how nonconservative forces bias random thermal fluctuations to induce circulating probability currents.

Figure 1 schematically represents the deceptively simple system. A single colloidal sphere is drawn to the focus of a converging laser beam by forces arising from gradients in the beam's intensity [1, 2]. These intensitygradient forces establish a three-dimensional potential energy well, $V(\boldsymbol{r})$, determined by the local intensity, $I(\boldsymbol{r})$. A particle trapped in this well also experiences radiation pressure that drives it downstream with a force proportional to $I(\boldsymbol{r})$. In the absence of thermal fluctuations, a trapped particle would come to rest at a stable mechanical equilibrium downstream of the focus.

Treating the displaced equilibrium point as the origin of an effective potential energy well is tempting but misleading. To appreciate the problem, consider a thermally driven trajectory such as the example shown schematically in Fig. 11. Were the system in thermodynamic equilibrium, forward and reverse trajectories around this loop would have equal probability. Because the light is more intense near the optical axis, however, radiation pressure biases the random walk in the forward direction. This departure from detailed balance should induce irreversible circulation in the particle's otherwise random fluctuations [4].

We demonstrate this effect by observing the motions of colloidal silica spheres $2.2 \mu \mathrm{m}$ in diameter (Bangs Lab-
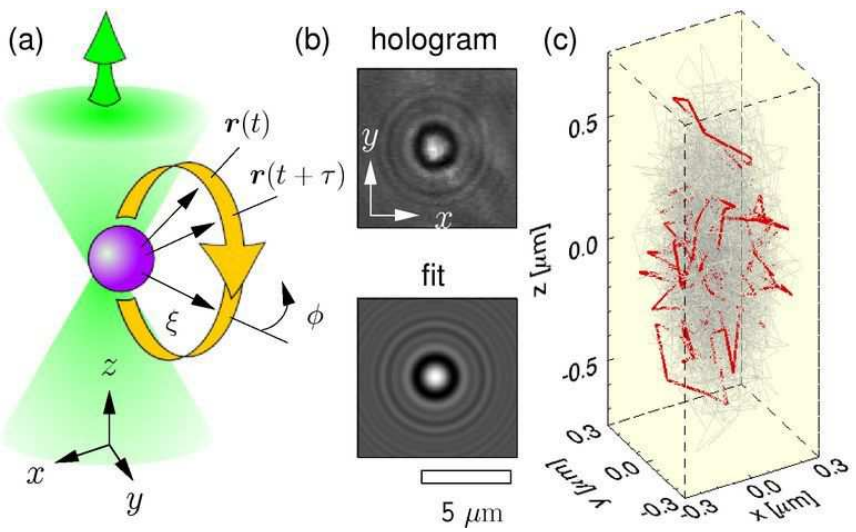

FIG. 1: (Color online) (a) Schematic representation of a colloidal sphere in an optical tweezer created from a beam of light propagating along $\hat{\boldsymbol{z}}$. The curving arrow represents a thermally driven trajectory. (b) Measured hologram of a trapped sphere together with a nonlinear least-squares fit to Mie scattering theory. (c) Measured 5 min trajectory in a trap at $P_{0}=7.5 \mathrm{~mW}$. Emphasized portion shows $10 \mathrm{~s}$.

oratories, SS04N/7651) dispersed in water and individually trapped in discrete optical tweezers. Each trap is formed from roughly $1 \mathrm{~mW}$ of laser light at a vacuum wavelength of $532 \mathrm{~nm}$ (Coherent Verdi) projected with the holographic optical trapping technique [5, 6, 6]. The beams are brought to a diffraction-limited focus by an objective lens (Nikon Plan Apo 100×, oil immersion) mounted in an inverted optical microscope (Nikon TE2000U). We track the particle in three dimensions with nanometer precision using video holographic microscopy [8, 9, 10]. In-line holograms, such as the example in Fig. 1(b), are created by illuminating the sphere with a collimated laser beam, in this case the $3 \mathrm{~mm}$ diameter beam provided by a $10 \mathrm{~mW}$ He-Ne laser (Uniphase) operating at a vacuum wavelength of $633 \mathrm{~nm}$. Light scattered by the particle interferes with the unscattered portion of the incident beam to produce an interference pattern in the microscope's focal plane. This is magnified and projected onto a CCD camera (NEC TI-324AII), which records holograms at video rates. Each frame in the video 


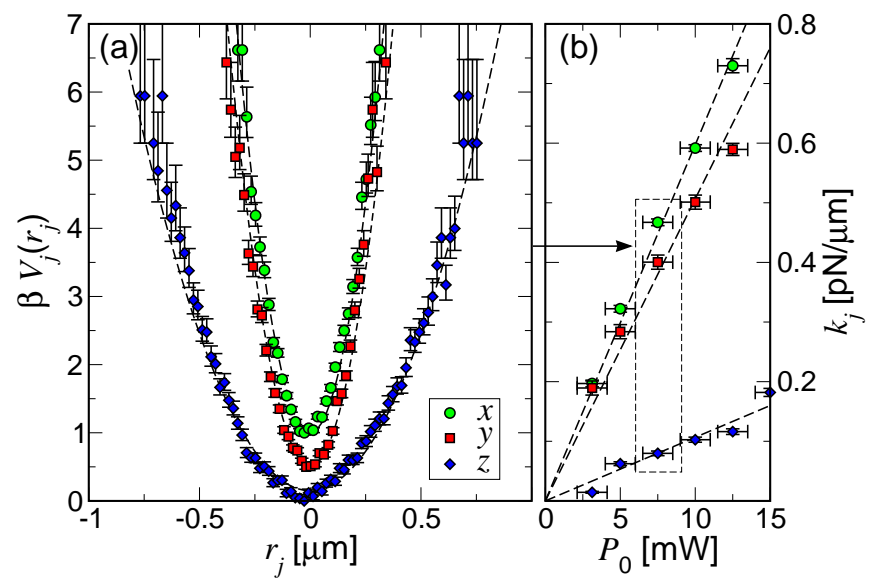

FIG. 2: (Color online) (a) Effective potential localizing a colloidal sphere to an optical trap at laser power $P_{0}=7.5 \mathrm{~mW}$, in units of the thermal energy scale $k_{B} T$. Dashed curves are parabolas whose curvatures yield the trap stiffness. Discrete points are obtained from histograms, $P_{j}\left(r_{j}\right)$, of the measured particle positions in $20 \mathrm{~nm}$ bins. The $x$ and $y$ results are offset by $1 k_{B} T$ and $0.5 k_{B} T$, respectively, for clarity. (b) Dependence of the effective trap stiffness on laser power.

stream then is fit to the predictions of Lorenz-Mie scattering theory [11] to obtain each particle's position in three dimensions, $\boldsymbol{r}(t)$, its radius $a$, and its complex index of refraction [10]. Figure 1(b) also shows the computed image resulting from fitting to the experimental image. A sequence of such fits yields the particle's threedimensional trajectory, as plotted in Fig. 1(c).

Were the particle to come to equilibrium within the trap, the probability to find it within $d \boldsymbol{r}$ of $\boldsymbol{r}$ would be given by the Boltzmann distribution,

$$
\rho(\boldsymbol{r})=N \exp (-\beta V(\boldsymbol{r})),
$$

where $\beta^{-1}=k_{B} T$ is the thermal energy scale at absolute temperature $T$ and $N$ is a normalization constant. This is the basis for a popular method to calibrate optical traps 12], with measured particle positions being compiled into $P(\boldsymbol{r})$, which then is inverted to yield $V(\boldsymbol{r})$. In the further approximation that the trap can be modeled as a three-dimensional harmonic well centered at the origin, the three-dimensional probability density can be factored into one-dimensional contributions, $\rho(\boldsymbol{r})=\prod_{j=1}^{3} N_{j} \exp \left(-\beta V_{j}\left(r_{j}\right)\right)$, where

$$
V_{j}\left(r_{j}\right)=\frac{1}{2} k_{j} r_{j}^{2}
$$

The trap is then characterized by an effective spring constant $k_{j}$ in each of the three Cartesian coordinates.

Figure 2(a) shows the result of applying Eq. (2) to 10,000 measurements of a single particle's position in an optical tweezer powered by $P_{0}=7.5 \pm 1.0 \mathrm{~mW}$ of light. The difference between the trap's apparent stiffness $k_{x}=0.467 \pm 0.009 \mathrm{pN} / \mu \mathrm{m}$ along the axis of the light's

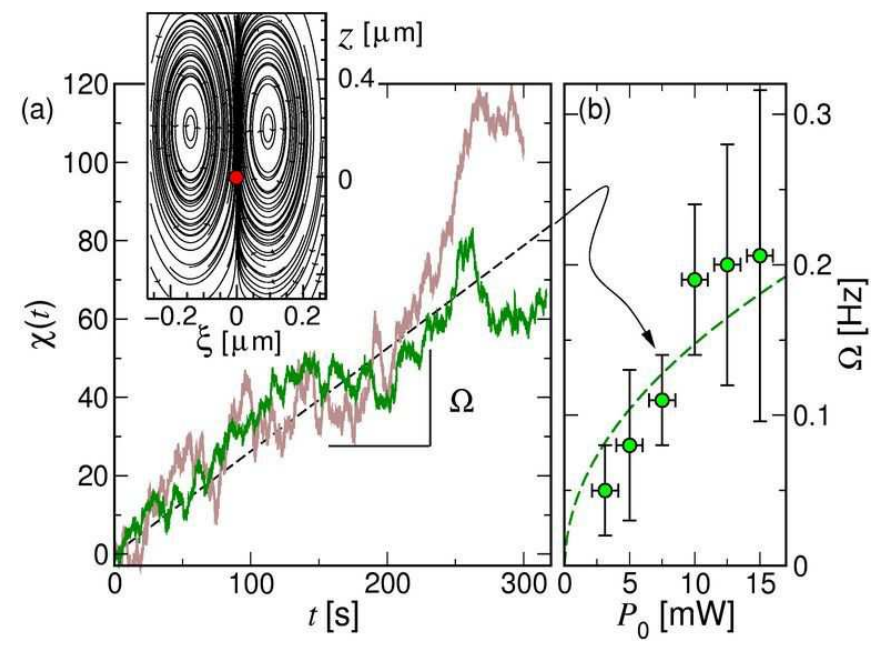

FIG. 3: (Color online) (a) Accumulated circulation of a single particle's trajectory over 10,000 time steps at laser power $P_{0}=7.5 \mathrm{~mW}$. Dark (green) trace: experimental data. Light (brown) trace: simulation results for equivalent conditions. Inset: streamlines of the measured velocity field in the $(\xi, z)$ plane, with the trap's center denoted by a circle. (b) Circulation rate as a function of laser power together with prediction of Eq. (12).

polarization and $k_{y}=0.400 \pm 0.008 \mathrm{pN} / \mu \mathrm{m}$ in the perpendicular direction is consistent with previous reports of polarization effects in optical trapping [13, 14, 15]. The axial stiffness, $k_{z}=0.080 \pm 0.004 \mathrm{pN} / \mu \mathrm{m}$ is a factor of 5 smaller because of the comparatively weak axial intensity gradients [16]. The dependence on laser power plotted in Fig. 2(b) confirms that $k_{j} \propto P_{0}$.

To quantify nonequilibrium effects in the particle's dynamics, we consider its trajectory in cylindrical coordinates, $\boldsymbol{r}=(\xi, \phi, z)$, centered on the trap's equilibrium position. During its thermally-driven exploration of the trap, the particle covers a roughly elliptical region in the $(\xi, z)$ plane. A statistical bias due to radiation pressure along the axis should appear as a tendency of the particle's trajectory to wind clockwise in the $(\xi, z)$ plane. To quantify this, we define a measure of the trajectory's circulation over the $\tau=1 / 30 \mathrm{~s}$ the interval between video frames,

$$
\Omega(t) \equiv \frac{1}{2 \pi} \frac{(\boldsymbol{r}(t+\tau) \times \boldsymbol{r}(t)) \cdot \hat{\boldsymbol{\phi}}}{\sqrt{\left\langle(\xi-\langle\xi\rangle)^{2}\right\rangle\left\langle(z-\langle z\rangle)^{2}\right\rangle}},
$$

which is positive for clockwise circulation and negative for retrograde motions. The angle brackets in Eq. (3) denote an average over the trajectory. To identify trends in $\Omega(t)$ despite large thermal fluctuations, we further define

$$
\chi(t) \equiv \int_{0}^{t} \Omega\left(t^{\prime}\right) d t^{\prime}
$$

which measures the accumulation of clockwise circulation in the particle's trajectory. The increasing trend in $\chi(t)$ 
confirms the existence of a circulating steady state in which the particle completes one net cycle in roughly $9 \mathrm{~s}$ with a mean drift speed of roughly $150 \mathrm{~nm} / \mathrm{s}$.

The measured circulation rate increases with laser power, as shown in Fig. 3(b). The particle also becomes increasingly well localized as the trap stiffens, however, so that the range of experimentally accessible laser powers is limited to $P_{0}<15 \mathrm{~mW}$ by our instrumental resolution.

Observing a convective flux in the trapped particles' trajectories confirms the system's departure from equilibrium. The nature of the nonequilibrium state is clarified by considering an idealized model of the system. The probability flux induced by a force $\boldsymbol{F}(\boldsymbol{r})$ acting on a Brownian particle is

$$
\boldsymbol{S}(\boldsymbol{r})=\mu \rho \boldsymbol{F}+D \nabla \rho,
$$

where $\rho(\boldsymbol{r})$ is the ensemble-averaged probability density for finding the particle near $\boldsymbol{r}$. We assume that the system reaches steady state so that $\rho(\boldsymbol{r})$ does not depend on time. Continuity of the probability density $(\nabla \cdot S=0)$ then yields the Fokker-Planck equation

$$
D \nabla^{2} \rho=\mu \boldsymbol{F} \cdot \nabla \rho+\mu \rho \nabla \cdot \boldsymbol{F} .
$$

The divergence-free flux still can support circulation,

$$
\nabla \times \boldsymbol{S}=\mu \boldsymbol{F} \times \nabla \rho+\mu \rho \nabla \times \boldsymbol{F}
$$

provided $\nabla \times \boldsymbol{F} \neq 0$.

We model the trap as a radially symmetric harmonic well with radiation pressure directed along the optical axis with a strength proportional to the local intensity:

$$
\boldsymbol{F}(\boldsymbol{r})=-k \boldsymbol{r}+f_{1} \exp \left(-\frac{r^{2}}{2 \sigma^{2}}\right) \hat{\boldsymbol{z}} .
$$

This model is parametrized the trap's stiffness, $k$, and the scale of radiation pressure, $f_{1}$, both of which are proportional to the laser's power, $P_{0}$. and also by the effective width of the trap, $\sigma$, which depends on the quality of the focus and the size of the trapped particle [17, 18, 19]. For a particle whose radius $a$ is substantially larger than the wavelength of light, $\sigma \gtrsim a$. Stable trapping requires the restoring force to exceed radiation pressure, so that $\epsilon=f_{1} /(k \sigma)$ may be taken to be small parameter independent of laser power. In that case, $\boldsymbol{F}(\boldsymbol{r})$ has a point of stable mechanical equilibrium at $z=\epsilon \sigma$.

The lighter trace in Fig. 3(a) shows the circulation observed in a fourth-order Runge-Kutta Brownian dynamics simulation of a particle diffusing in the force field described by Eq. (8), using parameters obtained from Fig. 2. and assuming $\epsilon \approx 0.1$. Both the slope of $\chi(t)$ and the magnitude of its fluctuations are consistent with experimental results. This agreement both supports our interpretation of our experimental observations and also indicates that Eq. (8) is sufficiently detailed for quantitative comparisons with our measurements.
Expanding the probability density to first order in $\epsilon$, $\rho(\boldsymbol{r})=\rho_{0}(r)+\epsilon \rho_{1}(\boldsymbol{r})$, yields an approximate solution [20] to Eq. (6),

$$
\begin{aligned}
& \rho_{0}(r)=\left(\frac{\beta k}{2 \pi}\right)^{\frac{3}{2}} \exp \left(-\frac{1}{2} \beta k r^{2}\right) \quad \text { and } \\
& \rho_{1}(\boldsymbol{r}) \approx \beta k \sigma \exp \left(-\frac{r^{2}}{2 \sigma^{2}}\right) z \rho_{0}(r) .
\end{aligned}
$$

Equation (9) is the result that would be obtained for a particle at equilibrium in a harmonic potential. Equation (10) describes the lowest-order correction due to spatially nonuniform radiation pressure. Because this expression for $\rho_{1}(\boldsymbol{r})$ is obtained in the stable-trapping limit, $\beta k \sigma^{2} \gg 1$, it underestimates the probability for the particle to make large-scale excursions from the trap. The results that follow therefore are conservative underestimates for the influence of non-conservative optical forces on a trapped particle's dynamics.

Even if the particle were to reach equilibrium in the force field $\boldsymbol{F}(\boldsymbol{r})$, the distortion of $\rho_{0}(r)$ by $\rho_{1}(\boldsymbol{r})$ would affect measurements of colloidal forces calibrated by thermal fluctuation analysis. The first-order correction not only displaces the center of the probability distribution downstream, but also broadens it. Consequently, the effective trap stiffness estimated from a probe particle's unloaded fluctuations will systematically underestimate the forces exerted by the trap under load, when fluctuations are suppressed.

Our experimental results demonstrate, furthermore, that the distorted probability density does not come to equilibrium, but rather undergoes steady-state circulation. Expanding $\boldsymbol{S}(\boldsymbol{r})$ to lowest non-vanishing order in $\epsilon$, we obtain the mean circulation rate [20]

$$
\begin{aligned}
\Omega_{0} & =\frac{1}{2 \pi} \int(\nabla \times \boldsymbol{S}) \cdot \hat{\boldsymbol{\phi}} d^{3} r \\
& \approx \frac{1}{\sqrt{18 \pi}} \frac{\mu f_{1}}{\sigma} \frac{\left(\beta k \sigma^{2}\right)^{\frac{1}{2}}}{1+\beta k \sigma^{2}} .
\end{aligned}
$$

This result can be generalized for an anisotropic trap by appropriately scaling the Cartesian coordinates in Eq. (8). In that case, Eq. (12) still holds with the effective spring constant set by the harmonic mean, $k=$ $3 /\left(k_{x}^{-1}+k_{y}^{-1}+k_{z}^{-1}\right)$. Given the results from Fig. 2, the measured circulation rates in Fig. 3(b) agree well with the predictions of Eq. (12) for $\epsilon=0.11 \pm 0.04$, including the predicted $P_{0}^{\frac{1}{2}}$ dependence on laser power.

Equation (12) also predicts that the circulation rate scales with temperature as $\Omega_{0} \sim T^{\frac{1}{2}}$ for $\beta k \sigma^{2}>1$. This confirms that the particle would not circulate at all were it not for thermal fluctuations. The optically trapped particle therefore is an exceptionally simple example of a Brownian motor 21, 22, 23 whose ability to perform work relies on rectification of thermal noise. Unlike previous optical implementations of thermal ratchets 
[24, 25, 26, 27, 28], this fountain of probability requires no time-dependent driving, but rather is biased into motion by the non-conservative component of the optical force [3].

The fountain's efficiency as a Brownian motor can be estimated by computing the power dissipated into the water by viscous drag given the particle's mean drift velocity, $\boldsymbol{v}(\boldsymbol{r})=\boldsymbol{S}(\boldsymbol{r}) / \rho(\boldsymbol{r})$ [29]. The result [20],

$$
P=\int \frac{|\boldsymbol{S}|^{2}}{\mu \rho} d^{3} r \approx 5 \mu f_{1}^{2} \frac{\left(\beta k \sigma^{2}\right)^{\frac{3}{2}}}{\left(2+\beta k \sigma^{2}\right)^{\frac{7}{2}}}
$$

depends strongly on laser power at low powers but becomes independent of $P_{0}$ in the strong-trapping limit. The maximum dissipation rate, $P_{\max }=5 \mu \epsilon^{2}\left(k_{B} T\right)^{2} / \sigma^{2}$, is a fixed proportion of the particle's thermally-driven velocity fluctuations. The system transduces fluctuations into heat through the bias imposed by the nonconservative optical force. Its efficiency is limited by the material properties that determine $\epsilon$, and thus by the requirement that the particle remain trapped.

Although this model is consistent with our measurements, other mechanisms also might cause a particle to circulate in an optical trap. For example, localized heating due to absorption of light at the focal point could create a toroidal convection roll that would advect the particle. This has been demonstrated with infrared optical tweezers operating at $1480 \mathrm{~nm}$ [30]. The optical absorption coefficient of water is five orders of magnitude smaller at $532 \mathrm{~nm}$, however 31. Scaling the previously reported [30] convection rate accordingly suggests that the maximum drift speed due to thermal convection in our system should be no greater than $1 \mathrm{~nm} / \mathrm{s}$ at the highest laser powers in out study. Thermal convection therefore appears unlikely to account for our observations.

If, indeed, steady-state circulation is an intrinsic feature of optically trapped particles' motions, the nonequilibrium effects we have identified should influence any measurement based on analysis of their thermal fluctuations. How such effects might have affected previously reported measurements remains to be determined. Establishing that a colloidal particle trapped in a static optical tweezer acts as a Brownian motor also creates new opportunities for research in nonequilibrium statistical physics. For example, the nonuniform intensity in an optical tweezer also exert position-dependent torques on the trapped particle that we have not considered here. The resulting nonequilibrium behavior in the particle's rotational degrees of freedom may share features in common with the fountain-like translational bias we have identified, and the two may be coupled in interesting ways. These considerations also may be extended to account for nonequilibrium effects in more general light fields.

We are grateful to Vincent Pereira and Alexander Grosberg for enlightening conversations. This work was supported by the National Science Foundation through
Grant Number DMR-0451589 and by a grant from Consolidated Edison.

[1] A. Ashkin, J. M. Dziedzic, J. E. Bjorkholm, and S. Chu, Opt. Lett. 11, 288 (1986).

[2] A. Ashkin, Biophys. J. 61, 569 (1992).

[3] Y. Roichman, B. Sun, Y. Roichman, J. Amato-Grill, and D. G. Grier, Phys. Rev. Lett. 100, 013602 (2008).

[4] K. Tomita and H. Tomita, Prog. Theor. Phys. 51, 1731 (1974).

[5] E. R. Dufresne and D. G. Grier, Rev. Sci. Instrum. 69, 1974 (1998).

[6] D. G. Grier, Nature 424, 810 (2003).

[7] M. Polin, K. Ladavac, S.-H. Lee, Y. Roichman, and D. G. Grier, Opt. Express 13, 5831 (2005).

[8] J. Sheng, E. Malkiel, and J. Katz, Appl. Opt. 45, 3893 (2006).

[9] S.-H. Lee and D. G. Grier, Opt. Express 15, 1505 (2007).

[10] S.-H. Lee, Y. Roichman, G.-R. Yi, S.-H. Kim, S.-M. Yang, A. van Blaaderen, P. van Oostrum, and D. G. Grier, Opt. Express 15, 18275 (2007).

[11] C. F. Bohren and D. R. Huffman, Absorption and Scattering of Light by Small Particles (Wiley Interscience, New York, 1983).

[12] E. L. Florin, A. Pralle, E. H. K. Stelzer, and J. K. H. Horber, Appl. Phys. A 66, S75 (1998).

[13] A. Rohrbach, Phys. Rev. Lett. 95, 168102 (2005).

[14] A. R. Zahkarian, P. Polynkin, M. Mansuripur, and J. V. Moloney, Opt. Express 14, 3660 (2006).

[15] R. S. Dutra, N. B. Viana, P. A. Maia Neto, and H. M. Nussenzveig, JOA 9 (2007).

[16] K. C. Vermeulen, G. J. L. Wuite, G. J. M. Stienen, and C. F. Schmidt, Appl. Opt. 45, 1812 (2006).

[17] K. Ladavac, K. Kasza, and D. G. Grier, Phys. Rev. E 70, 010901(R) (2004).

[18] M. Pelton, K. Ladavac, and D. G. Grier, Phys. Rev. E 70, 031108 (2004).

[19] Y. Roichman, V. Wong, and D. G. Grier, Phys. Rev. E 75, 011407 (2007).

[20] See EPAPS Document No. [number will be inserted by publisher] (2008), for details of the derivation of an optically trapped particle's circulation. For more information on EPAPS, see http://www . aip.org/pubservs/epaps.html

[21] P. Reimann, R. Bartussek, R. Haussler, and P. Hänggi, Phys. Lett. A 215, 26 (1996).

[22] P. Reimann, Phys. Rep. 361, 57 (2002).

[23] P. Reimann and P. Hänggi, Appl. Phys. A 75, 169 (2002).

[24] L. P. Faucheux, L. S. Bourdieu, P. D. Kaplan, and A. J. Libchaber, Phys. Rev. Lett. 74, 1504 (1995).

[25] L. P. Faucheux, G. Stolovitzky, and A. Libchaber, Phys. Rev. E 51, 5239 (1995).

[26] S.-H. Lee, K. Ladavac, M. Polin, and D. G. Grier, Phys. Rev. Lett. 94, 110601 (2005).

[27] S.-H. Lee and D. G. Grier, Phys. Rev. E 71, 060102(R) (2005).

[28] S.-H. Lee and D. G. Grier, J. Phys.: Condens. Matter 17, S3685 (2006).

[29] U. Seifert, Phys. Rev. Lett. 95, 040602 (2005).

[30] D. Braun and A. Libchaber, Phys. Rev. Lett. 89, 188103 
(2002).

[31] G. M. Hale and M. R. Querry, Appl. Opt. 12, 555 (1973). 